\title{
Effect of thermo-chemical treatments on the luminescence and scintillation properties of $\mathrm{CaWO}_{4}$
}

\author{
V. Yakovyna $^{\text {a,* }}$, Ya. Zhydachevskii' ${ }^{a}$, V.B. Mikhailik ${ }^{\text {b }}$, I. Solskii $^{c}$, D. Sugak ${ }^{c}$, M. Vakiv $^{\mathrm{c}}$ \\ ${ }^{a}$ Lviv Polytechnic National University, 12 Bandera, Lviv 79646, Ukraine \\ ${ }^{\mathrm{b}}$ Department of Physics, University of Oxford, Keble Road, Oxford OX1 3RH, UK \\ "Institute of Materials, SRC "Carat", 202 Stryjska, Lviv 79031, Ukraine
}

Received 10 September 2007; received in revised form 15 October 2007; accepted 7 November 2007

\begin{abstract}
In this work, we investigated the influence of thermal annealing in different atmospheres on the luminescence and scintillation properties of $\mathrm{CaWO}_{4}$. Luminescence and luminescence excitation spectra were examined using VUV synchrotron radiation. Thermal treatment causes change of the defect subsystem of the crystal, resulting in the alteration of the spectral dependences. The most obvious effect is the relative change in the intensity of the defect emission band which is excited below the fundamental absorption edge 4.7 eV. Studies of the scintillation kinetics and light yield carried out over a temperature range 8-300 K showed that former does not change upon the treatment, in contrast to the intensity of scintillations. It is found that $\sim 50 \%$ increase of the scintillation response can be achieved for $\mathrm{CaWO}_{4}$ samples annealed in hydrochloric acid.
\end{abstract}

(C) 2007 Elsevier B.V. All rights reserved.

PACS: $29.40 . \mathrm{Mc} ; 78.55 . \mathrm{Hx} ; 61.72 . \mathrm{Ji}$

\section{Introduction}

Among the tungstate crystals that are widely used as detectors of high-energy radiation calcium tungstate is one of the most promising materials due to its high light yield, currently assessed to be ca. $16,000 \mathrm{ph} / \mathrm{MeV}[1]$. However this parameter of $\mathrm{CaWO}_{4}$ can vary significantly, a characteristic associated with difference in the quality of samples. Given the high interest in the use of a scintillating $\mathrm{CaWO}_{4}$ in the experimental searches for rare events [2-5], the improvement of scintillation characteristic of the material is of primary importance.

The high-temperature annealing of complex oxide crystals in different atmospheres is an efficient tool to influence the defect subsystem of crystals. To get better insight into this effect in $\mathrm{CaWO}_{4}$, we investigated luminescence and

\footnotetext{
* Corresponding author. Tel.: +38 032 2582696; fax: +38 0322582153.

E-mail address: crystal@polynet.lviv.ua (V. Yakovyna).
}

scintillation properties of crystals subjected to various high-temperature treatments.

\section{Experimental}

Large $\mathrm{CaWO}_{4}$ crystals $(\varnothing 65 \mathrm{~mm}$, length of the cylindrical part $100 \mathrm{~mm}$ ) were grown by Czochralski technique in iridium crucibles under the flow of argon, with a small $(\sim 2 \%)$ content of oxygen. The crystals studied in the present work were $1 \mathrm{~mm}$ thick single crystalline plates cut from adjacent pieces of the same $\mathrm{CaWO}_{4}$ ingot ensuring identical optical properties. After polishing the samples were annealed in pure oxygen, nitrogen, hydrogen, vapours of hydrochloric acid or in vacuum. The annealing processes were performed in sealed quartz ampoules at temperatures varying from 600 to $1200{ }^{\circ} \mathrm{C}$. Each annealing process lasted $4 \mathrm{~h}$.

The luminescence studies were carried out using synchrotron radiation facilities at SUPERLUMI station (DESY, Hamburg) [6]. The excitation spectra of $\mathrm{CaWO}_{4}$ 
at room and $\mathrm{LHeT}$ in the region $4-8 \mathrm{eV}$ were monitored at the position of high $(2.9 \mathrm{eV})$ and low-energy $(2.3 \mathrm{eV})$ emission bands. The scintillation characteristics of the crystals were measured in the 8-300 $\mathrm{K}$ temperature range at particle excitation $\left({ }^{241} \mathrm{Am} \alpha\right.$-source $)$ using the multiple photon counting coincidence (MPCC) technique [7]. The technique allows measurements of the number of photons per scintillation event with the latter being proportional to the scintillator light yield. Because of this the effect of geometry on the measured light yield is significantly inhibited and different samples can be compared accurately.

\section{Results and discussion}

The emission spectra of $\mathrm{CaWO}_{4}$ samples under study are presented in Fig. 1a and b. The use of the photo-excitation at various wavelengths allowed to separate the high-energy
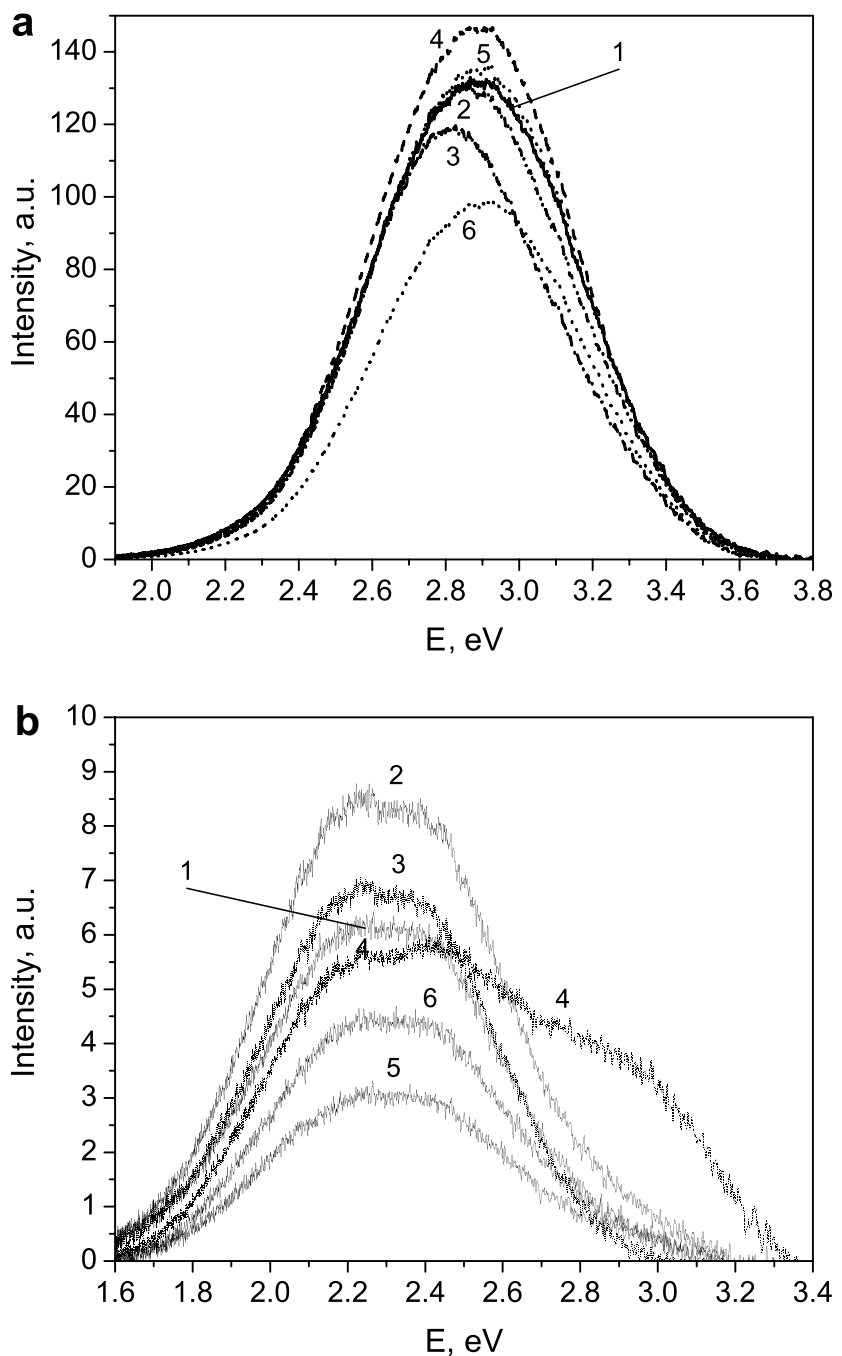

Fig. 1. The emission spectra of $\mathrm{CaWO}_{4}$ samples monitored using an optical excitation at $\lambda=230 \mathrm{~nm}(5.4 \mathrm{eV})$ (a) and $\lambda=280 \mathrm{~nm}(4.4 \mathrm{eV})$ (b). 1 - reference sample, 2 - annealed in oxygen atmosphere at $900{ }^{\circ} \mathrm{C}, 3-$ annealed in oxygen at $1200^{\circ} \mathrm{C}, 4-$ annealed in nitrogen atmosphere at $1200{ }^{\circ} \mathrm{C}, 5-$ annealed in hydrogen at $600{ }^{\circ} \mathrm{C}, 6$ - annealed in vacuum at $1200^{\circ} \mathrm{C}$. (blue) and low-energy (green) emission bands of the crystal. As it is seen from the figure, the maximum of the blue emission for samples annealed in oxygen and nitrogen is slightly shifted towards the low-energy side in comparison with samples annealed in hydrogen or vacuum. A maximum of the green emission band is located at $2.3 \mathrm{eV}$ for all the samples under study. An interesting feature was observed for the crystal annealed in nitrogen: under excitation at $\lambda=280 \mathrm{~nm}(4.4 \mathrm{eV})$ a significant blue emission component was observed for this crystal (Fig. 1b, curve 4).

It is generally accepted that the blue emission of tungstates with scheelite structure is due to the radiative decay of self-trapped excitons localized at the regular $\mathrm{WO}_{4}^{2-}$ complexes while the green emission is associated with anion defective $\mathrm{WO}_{3}$ complexes [8-11]. Fig. 2 shows the excitation spectra monitored at various emission wavelengths for the reference sample and a sample annealed in the nitrogen atmosphere. The excitation spectra of the blue emission monitored at $\lambda=380 \mathrm{~nm}(3.3 \mathrm{eV})$ for the samples
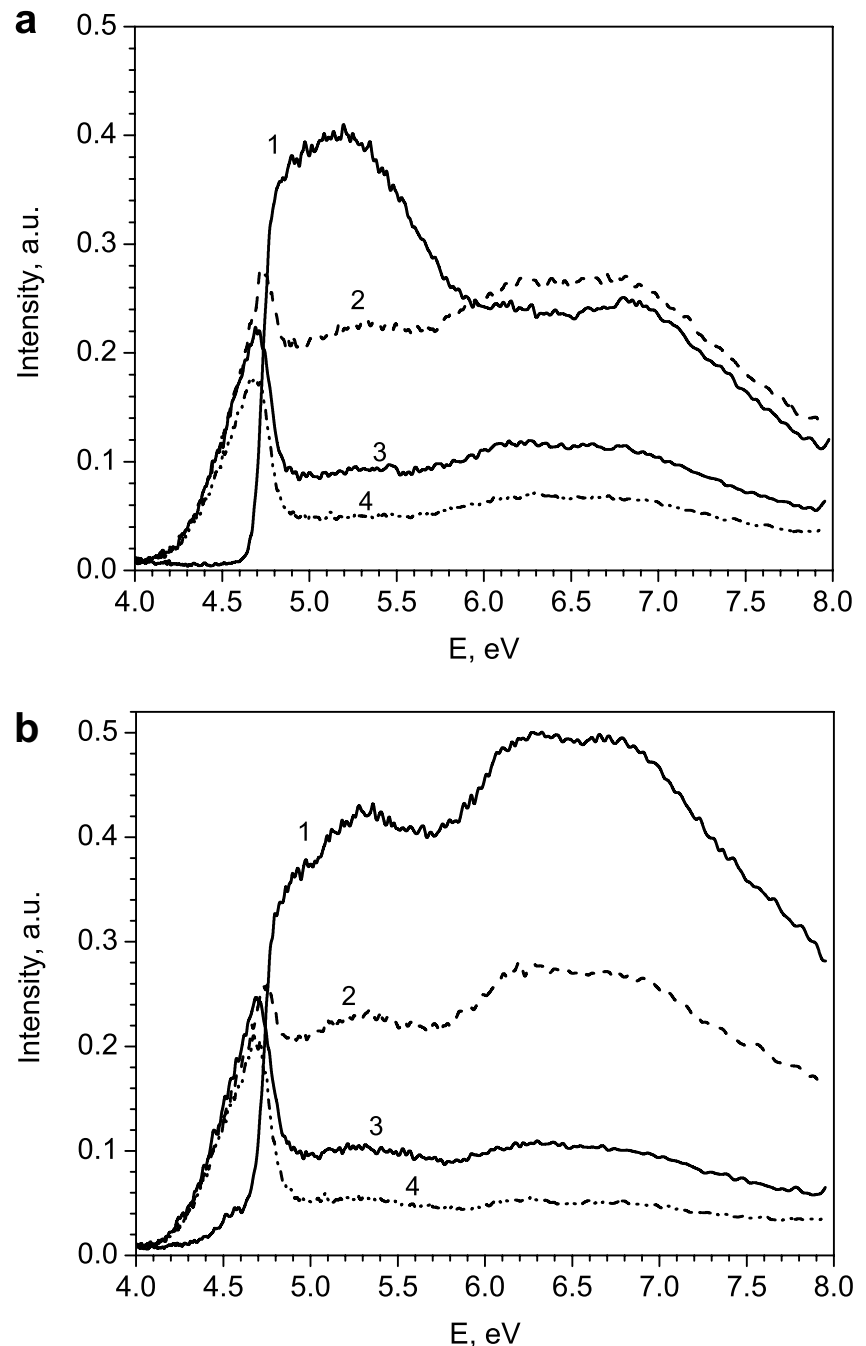

Fig. 2. The excitation spectra of the emission recorded at $\lambda=380 \mathrm{~nm}$ $(3.3 \mathrm{eV})(1), \lambda=520 \mathrm{~nm}(2.4 \mathrm{eV})(2), \lambda=550 \mathrm{~nm} \quad(2.3 \mathrm{eV}) \quad(3)$ and $\lambda=570 \mathrm{~nm}(2.2 \mathrm{eV})(4)$ for the reference sample (a) and sample annealed in nitrogen atmosphere at $1200^{\circ} \mathrm{C}(\mathrm{b})$. 


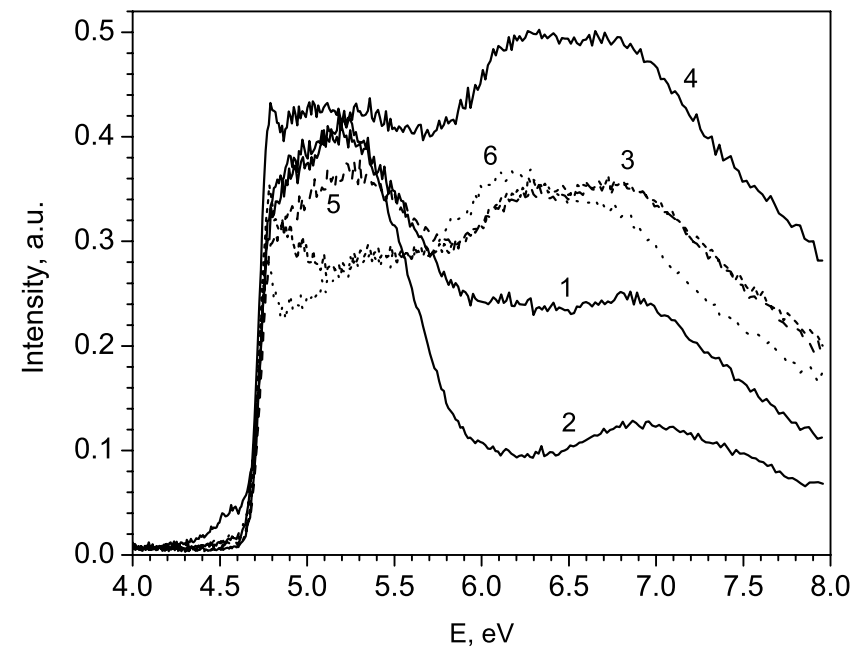

Fig. 3. The excitation spectra of the blue emission recorded at $\lambda=380 \mathrm{~nm}$ $(3.3 \mathrm{eV}) .1$ - reference sample, 2 - annealed in oxygen atmosphere at $900{ }^{\circ} \mathrm{C}, 3$ - annealed in oxygen at $1200{ }^{\circ} \mathrm{C}, 4-$ annealed in nitrogen atmosphere at $1200^{\circ} \mathrm{C}, 5-$ annealed in hydrogen at $600{ }^{\circ} \mathrm{C}, 6-$ annealed in vacuum at $1200^{\circ} \mathrm{C}$.

annealed in different conditions are shown in Fig. 3. The common feature of the excitation spectra of the blue emission is an onset at $4.7 \mathrm{eV}$ which is associated with the fundamental absorption edge, and two bands spanning the range $4.7-5.5 \mathrm{eV}$ and $6-8 \mathrm{eV}$. Given that the energy gap of $\mathrm{CaWO}_{4}$ is estimated to be $5.2 \mathrm{eV}$ [10], we infer that the low-energy band is due to the excitation of correlated electron-hole pairs that can form excitons [12]. The highenergy bands can be interpreted on the basis of calculation of $\mathrm{CaWO}_{4}$ energy structure $[13,14]$ as charge-transfer transitions inside $\mathrm{WO}_{4}^{2-}$ complex from the $\mathrm{O} 2 \mathrm{p}$ valence band to different sub-bands of W5 $\mathrm{d}$ conduction band. In contrast to the blue emission, the green luminescence is excited mainly in the narrow range of 4.2-4.7 eV near to the fundamental absorption edge. This observation agrees well with the energy scheme of defective $\mathrm{WO}_{3}$ complexes in tungstates with a sheelite structure that exhibit an excitation edge shifted toward the low-energy region $[15,16]$.

The excitation spectra of the blue emission of the samples annealed in different conditions differs by the intensity ratio of the excitonic transitions $(4.7-5.5 \mathrm{eV})$ and the charge-transfer transitions of $\mathrm{WO}_{4}^{2-}$ complexes $(6-8 \mathrm{eV})$. Furthermore the intensity ratio of the components in the 6-8 eV region is also different for the different samples (see Fig. 3). This is likely due to the change of the energy structure of the regular $\mathrm{WO}_{4}$ complexes under the influence of defects in the anion sublattice, such as interstitial oxygen or oxygen vacancies. It should be noted, that the oxygen annealing at various temperatures results in opposite effect on the structure of excitation spectra (curves 2 and 3 in Fig. 3). This fact can be connected with a two stage process of oxygen incorporation into $\mathrm{CaWO}_{4}$ crystal [17]. It is also noticeable that annealing in hydrogen and vacuum caused the same effect as the annealing in oxygen at $1200{ }^{\circ} \mathrm{C}$.
The excitation spectrum of the sample annealed in nitrogen should be mentioned separately. In this case the contribution of the $6-8 \mathrm{eV}$ band strongly increases. Besides, one can see a shoulder below the fundamental absorption edge at $4.2-4.7 \mathrm{eV}$ (see curve 4 in Fig. 3). This excitation shoulder leads to the appearance of the blue emission at lowenergy excitation of the crystal (see Fig. 1b, curve 4). We explain this observation by incorporation of nitrogen into the crystal and elimination of oxygen vacancies that are present in the crystal structure. Because of this the tungsten oxidation state changes from $6+$ to $5+$, creating the energy levels near the top of the valence band.

The temperature dependences of the decay time constants of fast and slow scintillation components are presented in Fig. 4. They were obtained by fitting experimental decay curves using a sum of two exponents. Interpretation of excited state dynamics in tungstate crystals is based on the energy scheme of emission complex that involves one ground and two excited states the lower of which one is metastable $[9,18]$. The temperature dependence of the decay time constant can be approximated by an equation that presents the decay time shortening as a result of a thermally activated re-population of a excited states and their non-radiative de-excitation [19].

$\frac{1}{\tau}=\frac{k_{1}+k_{2} \exp (-D / k T)}{1+\exp (-D / k T)}+K \exp (-\Delta E / k T)$

Here $k_{2}$ and $k_{1}$ are probabilities of radiative decay from the upper and lower excited states to the ground state respectively, $D$ is the energy distance between the excited states. The decay rate $k$ and the energy barrier $\Delta E$ are the characteristic parameters of the non-radiative luminescence quenching process.

The first term of Eq. (1) describes the decay time shortening at temperatures above $20 \mathrm{~K}$, while the second term is responsible for its decrease at $T>200 \mathrm{~K}$. The fit of the

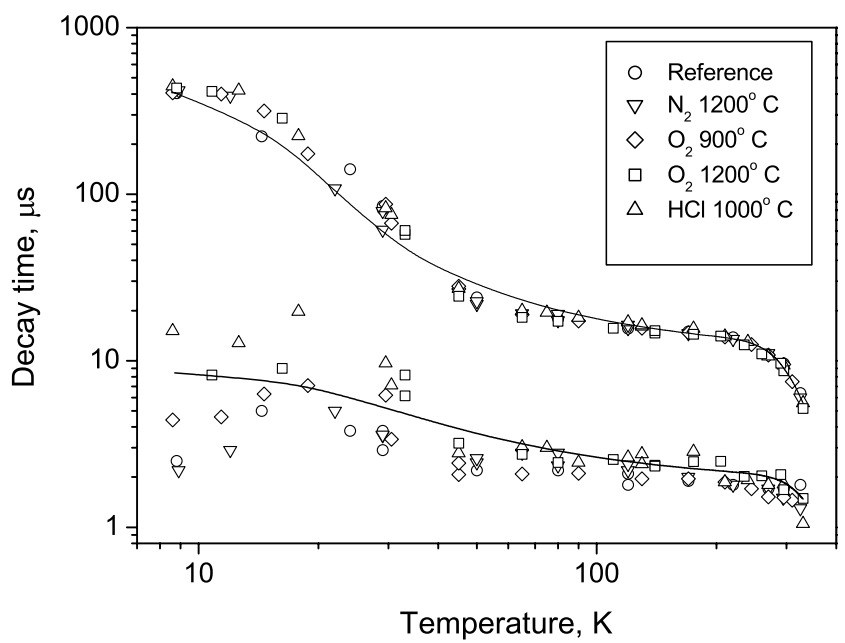

Fig. 4. Temperature dependence of the fast and slow scintillation decay times measured at particle excitation $\left({ }^{241} \mathrm{Am} \alpha\right.$-source $)$ for $\mathrm{CaWO}_{4}$ samples annealed at different conditions, and their theoretical approximation (see text for details). 
experimental data to this equation gives $D \sim 5 \mathrm{meV}$ and $\Delta E \sim 300 \mathrm{meV}$ for both fast and slow components that are consistent with values obtained earlier [18-20]. It is obvious that the decrease of decay time constant above $200 \mathrm{~K}$ is due to the thermal quenching of luminescence because of non-radiative transitions. The change of the decay time constant below $20 \mathrm{~K}$ is due to the presence of the metastable level below the emitting level that starts to play major role at very low temperatures.

The temperature dependence of integrated light yield for samples annealed in different conditions are shown in Fig. 5. One can distinguish three characteristic segments in these dependences: the region of the thermal quenching above $230 \mathrm{~K}$, a plateau region $(30-230 \mathrm{~K})$ where small changes of the light yield are observed and the low temperature region below $30 \mathrm{~K}$ where light yield exhibits rapid increase and than drops. It has to be noted that observed decrease of the light yield represents an experimental artefact: significant increase of the scintillation decay time at the fixed record length leads to the situation where a certain fraction of the emitted photons escape detection. When being corrected for this effect, the light shows no decrease in this range (see [7] for details). Of practical importance is that the observed temperature dependencies for samples subjected to different treatments run in parallel. This allows use of the results of the light yield measured at room temperature as indicative for the assessment of this parameter at low temperature while analysing the effect of different treatments.

From Fig. 5 one can notice that the light yield of the samples annealed in oxygen at 900 and $1200{ }^{\circ} \mathrm{C}$ as well as in air is somewhat lower than that of the reference (untreated) sample. This decrease of light yield can be understood by taking into account that the emission intensity of $\mathrm{CaWO}_{4}$ subjected to thermal treatment depends on the partial pressure of oxygen and exhibits maximum at

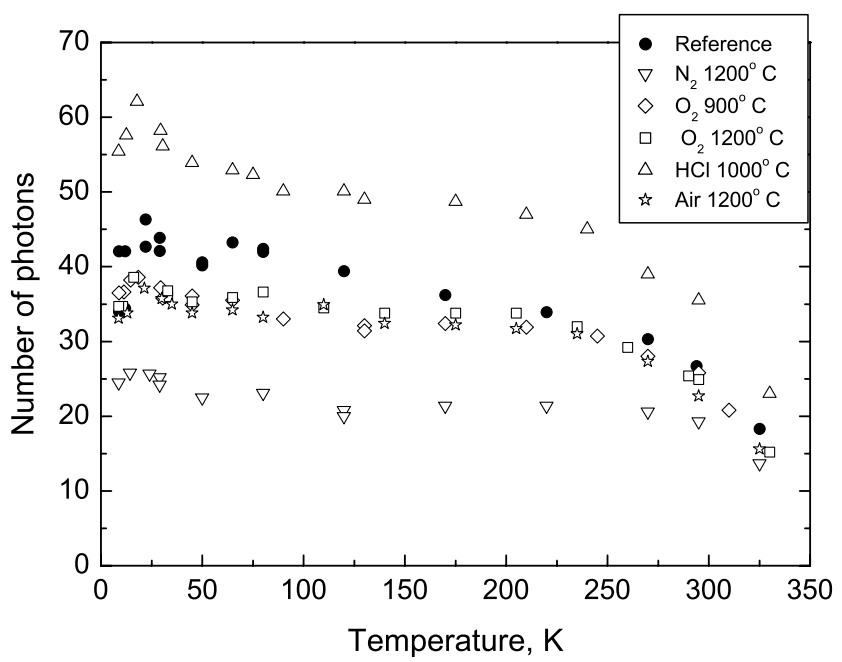

Fig. 5. Temperature dependence of the light yield measured at particle excitation $\left({ }^{241} \mathrm{Am} \alpha\right.$-source $)$ for the $\mathrm{CaWO}_{4}$ samples annealed at different conditions. $p \sim 10^{-4} \mathrm{~Pa}[21]$. Given that this parameter was not controlled in our experiment it is most likely that it is far from optimum, resulting in excessive formation of defect centers. The light yield of the sample annealed in nitrogen is significantly inhibited. We suppose that nitrogen being incorporated in the crystal replace oxygen vacancies and forms acceptor levels in the band gap. This facilitates non-radiative deactivation of the excited states.

The most interesting result is a substantial increase of the light yield from the crystal annealed in $\mathrm{HCl}$ vapour. Chemical etching is known to be helpful in improving the performance characteristics of scintillating crystals [2224]. It gives a rough surface finish comparing with the mechanical polish [22] improving the light collection in the readout configuration when there is no optical contact between the sample and detector [24]. In this respect the rough finish is identical to the diffused surface since it creates beneficial conditions for the trapped light to escape the crystal.

\section{Conclusions}

Our experiments revealed significant influence of hightemperature annealing at different conditions on the luminescence and scintillation properties of calcium tungstate crystals. The high energy part of the luminescence excitation spectra $(6-8 \mathrm{eV})$ which is attributed to the chargetransfer transitions in the $\mathrm{WO}_{4}^{2-}$ oxyanion complex exhibits very significant changes in the course of such treatment. The annealing conditions have a tangible effect on the intensity and spectral composition of the $\mathrm{CaWO}_{4}$ emission. The spectral changes are due to a variation of the relative intensity of the green emission caused by defects. The scintillation decay time constants and the light yield were measured over the temperature range $8-350 \mathrm{~K}$ and no change in the kinetics of the scintillation due to the thermal treatment were observed. On the other hand, changes of defect subsystem of the crystal induced by thermal treatment lead to the noticeable changes in the light yield. The possibility of a substantial $(\sim 50 \%)$ increase of the scintillation response using treatment of the $\mathrm{CaWO}_{4}$ crystals in hydrochloric acid is demonstrated. It is expected that the results of this study should be helpful for the optimization of production of $\mathrm{CaWO}_{4}$ crystals with improved scintillation characteristics.

\section{Acknowledgements}

The work was partially supported by the Ukrainian Ministry of Education and Science (project DB/Segnet) and the grant from the Royal Society (London). The studies at HASYLAB were supported by the European Community - Research Infrastructure Action under the FP6 "Structuring the European Research Area" Program through the Integrated Infrastructure Initiative "Integrating Activity on Synchrotron and Free Electron Laser Science". 


\section{References}

[1] M. Moszyński, M. Balcerzyk, W. Czarnacki, et al., Nucl. Instr. Meth. Phys. Res. A 553 (2005) 578.

[2] C. Cozzini, G. Angloher, C. Bucci, et al., Phys. Rev. C 70 (2004) 064606.

[3] G. Angloher, C. Bucci, P. Christ, et al., Astropart. Phys. 23 (2005) 325.

[4] V.B. Mikhailik, H. Kraus, J. Phys. D: Appl. Phys. 39 (2006) 1181.

[5] Yu.G. Zdesenko, F.T. Avignone III, V.B. Brudanin, et al., Astropart. Phys. 23 (2005) 249.

[6] G. Zimmerer, Nucl. Instr. Meth. Phys. Res. A 308 (1991) 178.

[7] H. Kraus, V.B. Mikhailik, D. Wahl, Nucl. Instr. Meth. Phys. Res. A 553 (2005) 522.

[8] R. Grasser, A. Sharmann, K.-R. Strack, J. Lumin. 27 (1982) 283.

[9] V. Mürk, M. Nikl, E. Mihokova, K. Nitsch, J. Phys. Condens. Matter 9 (1997) 249.

[10] V.B. Mikhailik, H. Kraus, D. Wahl, et al., Phys. Rev. B 69 (2004) 205110.

[11] V.B. Mikhailik, H. Kraus, G. Miller, et al., J. Appl. Phys. 97 (2005) 083523.
[12] V.V. Mikhailin, A.N. Belsky, I.A. Kamenskikh, et al., Nucl. Instr. Meth. Phys. Res. A 488 (2002) 367.

[13] Y. Zhang, N.A.W. Holzwarth, R.T. Williams, Phys. Rev. B 57 (1998) 12378.

[14] M. Itoh, N. Fujita, Y. Inabe, J. Phys. Soc. Jpn. 75 (2006) 084705.

[15] A.A. Annenkov, M.V. Korzhik, P. Lecoq, Nucl. Instr. Meth. Phys. Res. A 490 (2002) 30.

[16] Yu.A. Hizhnyi, S.G. Nedilko, J. Lumin. 102-103 (2003) 688.

[17] V. Yakovyna, A. Matkovskii, D. Sugak, et al., Radiat. Meas. 38 (2004) 401.

[18] G. Blasse, G. Bokkers, J. Solid State Chem. 49 (1983) 126.

[19] V.B. Mikhailik, H. Kraus, S. Henry, A.J.B. Tolhurst, Phys. Rev. B 75 (2007) 184308.

[20] G.B. Beard, W.H. Kelly, M.L. Mallory, J. Appl. Phys. 33 (1962) 144.

[21] B.M. Sinel'nikov, E.V. Sokolenko, E.G. Zvekova, Inorg. Mater. 32 (1996) 661.

[22] K. Kurashige, Y. Kurata, H. Ishibashi, K. Susa, IEEE Trans. Nucl. Sci. 45 (1998) 522.

[23] J.S. Huber, W.W. Moses, M.S. Andreaco, et al., Nucl. Instrum. Meth. A 437 (1999) 374.

[24] D. Strul, J. Sutcliffe-Goulden, P. Halstead, P.K. Marsden, IEEE Trans. Nucl. Sci. 49 (2002) 619. 